\title{
PRESENTATION OF RETINOBLASTOMA AS PHTHISIS BULBI
}

\author{
PAUL B. MULLANEY ${ }^{1}$, ZEYNEL A. KARCIOGLU ${ }^{1,2}$, SALEH AL-MESFER $^{1}$ \\ and EMAD B. ABBOUD ${ }^{1}$ \\ Riyadh, Saudi Arabia and New Orleans, Louisiana
}

\begin{abstract}
SUMMARY
Purpose: We sought to determine the incidence of retinoblastoma patients who presented with phthisis bulbi.

Methods: The medical records of 272 patients in the King Khaled Eye Specialist Hospital Retinoblastoma Registry were retrospectively studied. Clinical records, radiological investigations and histopathological slides were reviewed.

Results: We found that $2.7 \%$ of patients had retinoblastoma coincident with phthisis bulbi. Five of 10 patients had bilateral retinoblastoma; in the others it was unilateral. Radiologically, intraocular calcification was present in all except one case. All enucleated phthisical globes had residual viable tumour cells; optic nerve extension was found in 2 patients who had longstanding phthisis bulbi.

Conclusion: Phthisis bulbi is an uncommon presenting sign of retinoblastoma which often occurs after an ocular inflammatory episode possibly related to intraocular tumour infarction. In most cases the tumour is not visible because of intraocular disruption. That every enucleated eye in this series harboured well-differentiated tumour cells underlies the seriousness with which phthisis bulbi of unknown origin in children should be investigated for retinoblastoma.
\end{abstract}

Phthisis bulbi (PB) is a relatively uncommon presentation of retinoblastoma. The incidence is stated to be $2 \% .^{1,2}$ However, others have claimed that $\mathrm{PB}$ is not seen in association with retinoblastoma. $^{3}$

The cause of $\mathrm{PB}$ is thought to be occlusion of the central retinal vessels, which may lead to either partial or total regression of the intraocular tumour. $^{4-7}$ Trauma and infection are also common

From: ${ }^{1}$ King Khaled Eye Specialist Hospital, Riyadh, Saudi Arabia; ${ }^{2}$ Tulane University Medical School, New Orleans, Louisiana, USA.

Correspondence to: Dr Paul B. Mullaney, c/o Medical Library, King Khaled Eye Specialist Hospital, PO Box 7191, Riyadh 11462, Saudi Arabia. Fax: 011-966-482-1908 causes of PB. Because intraocular calcification is seen in $\mathrm{PB}$ and retinoblastoma, it may be difficult to ascertain whether a phthisical eye is harbouring retinoblastoma. This is particularly important if the tumour is only partially infarcted.

To establish the incidence amongst our population, we reviewed the clinical histories of all patients presenting to King Khaled Eye Specialist Hospital (KKESH) who were subsequently diagnosed with retinoblastoma. Those children who presented with or who developed PB are included in this report, and their clinical, radiological and histopathological findings are documented.

\section{PATIENTS AND METHODS}

The medical records of 272 patients in the KKESH Retinoblastoma Registry were reviewed and charts indicating a phthisical eye harbouring a retinoblastoma were retrieved. Ten records were included in the study. At some point, all patients in this series were clinically managed by one or more of the authors. The radiology material and the histopathology slides were also reviewed by one or more of the authors.

The clinical status on presentation was reviewed. In particular, the length of time leucocoria was present and any prior episode of endophthalmitis or cellulitis was noted. Corneal clarity, the status of the anterior chamber and presence or absence of cataract were documented. Also noted was media opacification which precluded a thorough posterior segment examination.

Computed tomographic (CT) examination had been performed in all but one patient. The films were reviewed, and the characteristics of the intraocular tumours were noted.

Histopathology slides were reviewed in 9 of 10 cases; no enucleation was done in case 10 . All slides had been processed with standard paraffin embedding and stained with haematoxylin and eosin

Eye (1997) 11, 403-408 C 1997 Royal College of Ophthalmologists 
Table I. Clinical and radiological findings in phthisical retinoblastoma eyes

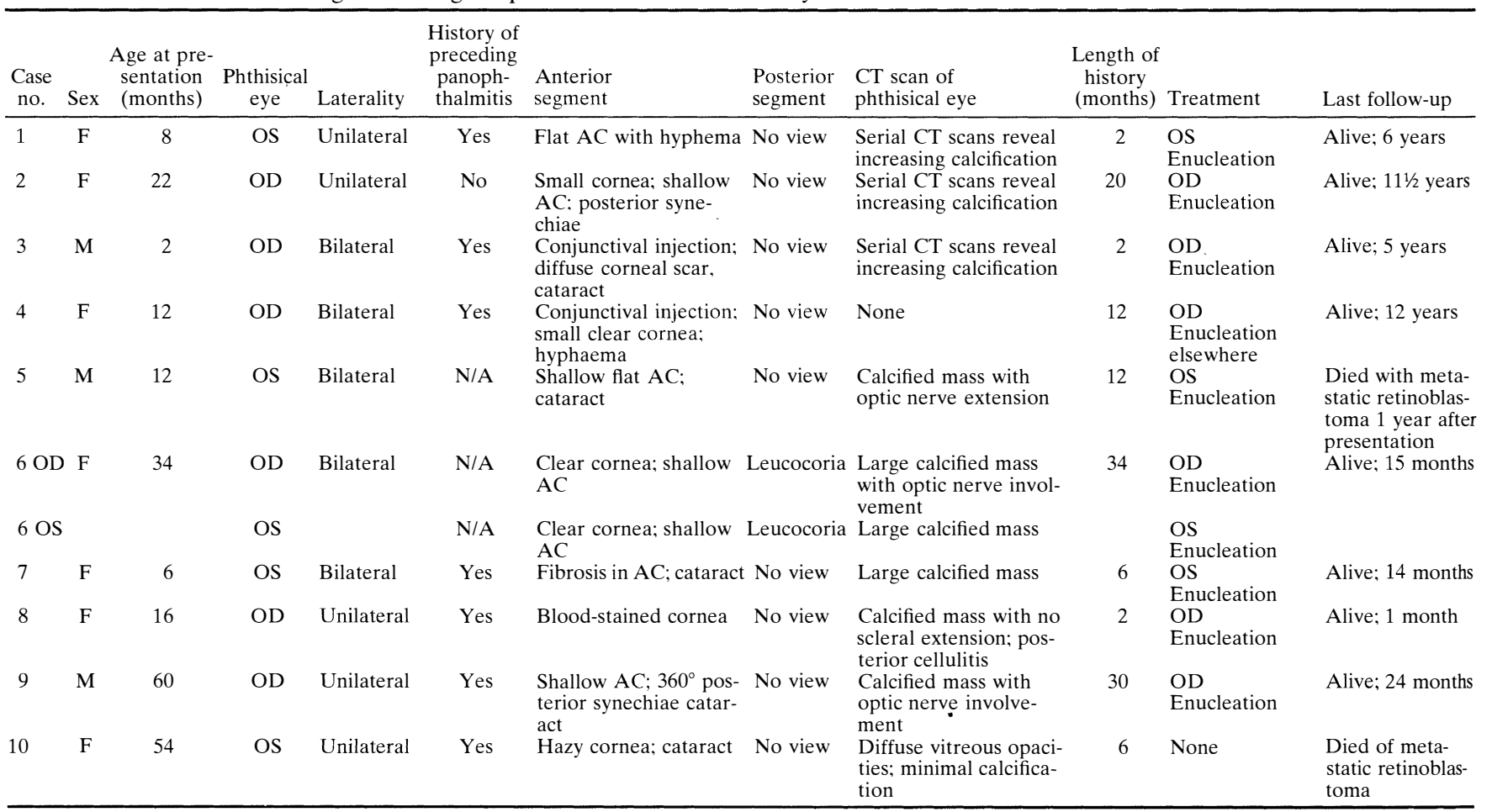

AC, anterior chamber; NA, not available.

(H\&E) and periodic acid-Schiff (PAS). The following histopathological parameters were established: the presence or absence of retinoblastoma, the degree of necrosis, calcification and ossification within the tumour. Involvement of the anterior chamber, choroid, sclera, optic nerve, subarachnoid space and orbit were expressed as a positive or negative status.

\section{RESULTS}

Nine patients presented with PB and an additional one developed it subsequently; 7 were female and 3 male. One patient had bilateral phthisis bulbi on presentation. The incidence of $\mathrm{PB}$ with retinoblastoma was $2.7 \%$. Age at presentation ranged between 2 and 60 months with a mean of 22.6 months. Five of the patients had bilateral retinoblastoma, and in 5 it was unilateral. In the patients with bilateral retinoblastoma, only the phthisical eye will be alluded to.
In the 8 patients in whom a history of preceding panophthalmitis was available, in 7 it was affirmative. All 7 had had medical treatment for presumed conjunctivitis or cellulitis. In all 7, PB was noted after the inflammatory episode. Corneal or lenticular opacification precluded any view of the posterior segment in 9 of 10 patients (Table I). CT scans were performed in the majority of patients $(9 / 10)$. A marked degree of intraocular calcification was seen in every case. In cases 1-3, serial CT scan examinations showed increasing calcification with progression to phthisis.

All patients except case 10 (parental refusal) underwent enucleation of the affected eye. Subsequent histopathological examination revealed viablelooking retinoblastoma cell populations in all eyes. As indicated in Table II, cataract formation was present in every lens examined. Necrosis and calcification were present to a large degree in all

Table II. Histopathological findings in phthisical retinoblastoma eyes ${ }^{\mathrm{a}}$

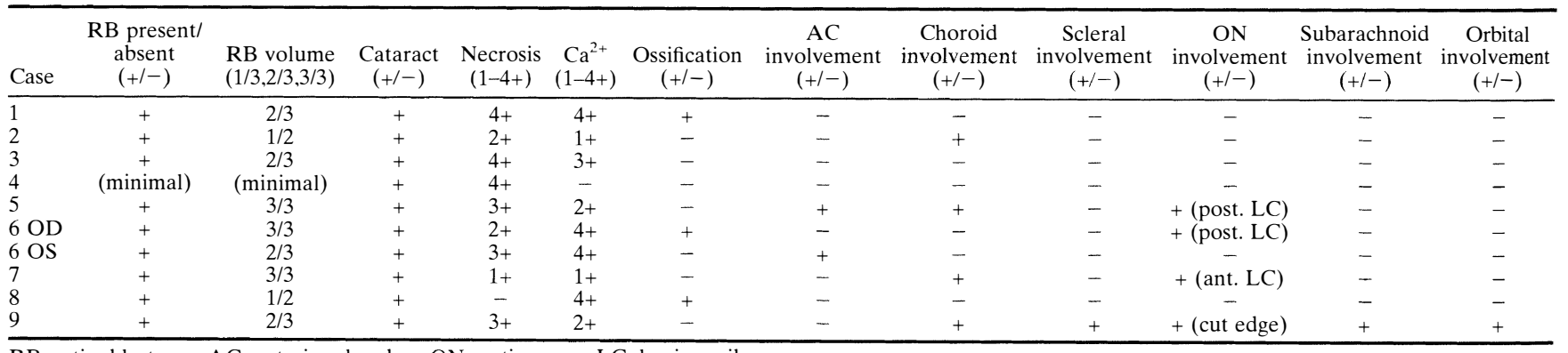

$\mathrm{RB}$, retinoblastoma; $\mathrm{AC}$, anterior chamber; $\mathrm{ON}$, optic nerve; LC, lamina cribrosa.

${ }^{\mathrm{a}}$ No enucleation was done in case 10 . 


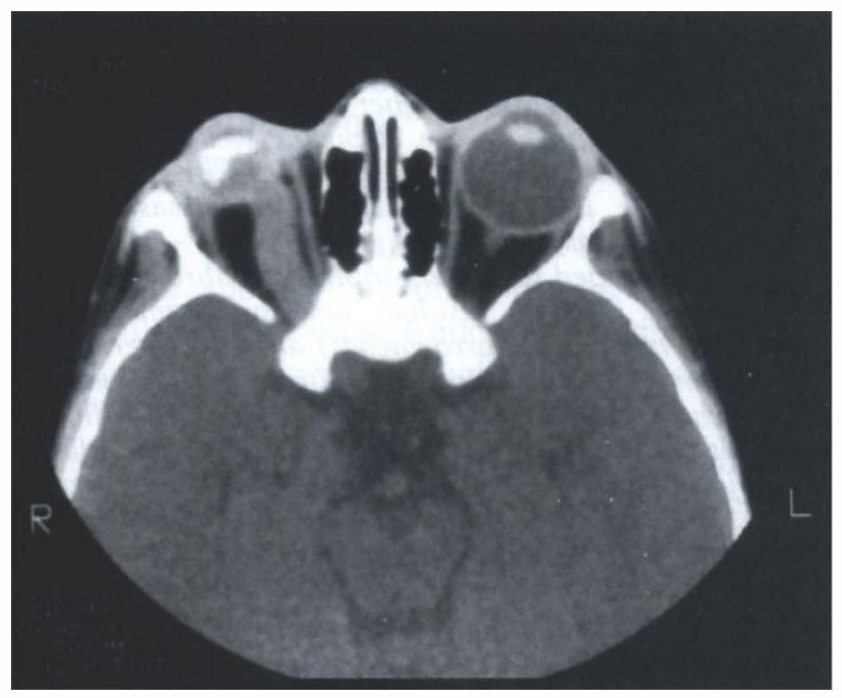

Fig. 1. CT scan of case 9 showing phthisis bulbi (PB) and markedly thickened right optic nerve.

globes examined. Focal choroidal involvement was seen in cases 2, 5, 7 and 9 (Table II). Optic nerve involvement was seen in the right eye of cases 6 and 9 (Fig. 1) and in the left eye of cases 5 and 7. Table I illustrates that $\mathrm{PB}$ was present for 30 months or longer in cases 6 and 9; both of these cases had the greatest extent of optic nerve involvement. Two patients (cases 5 and 10) in this series have died from disseminated metastases. In case 5 this originated from the contralateral eye.

\section{CASE REPORT}

A 6-month-old female infant (case 7) presented to KKESH with a shrinking left eye (Fig. 2). This eye was swollen and cellulitic shortly after birth. The infant was given topical and oral antibiotics after which shrinkage was noted. The right eye manifested leucocoria with a total retinal detachment which brought the retina immediately behind the lens. Clinical examination of the left eye showed PB. The

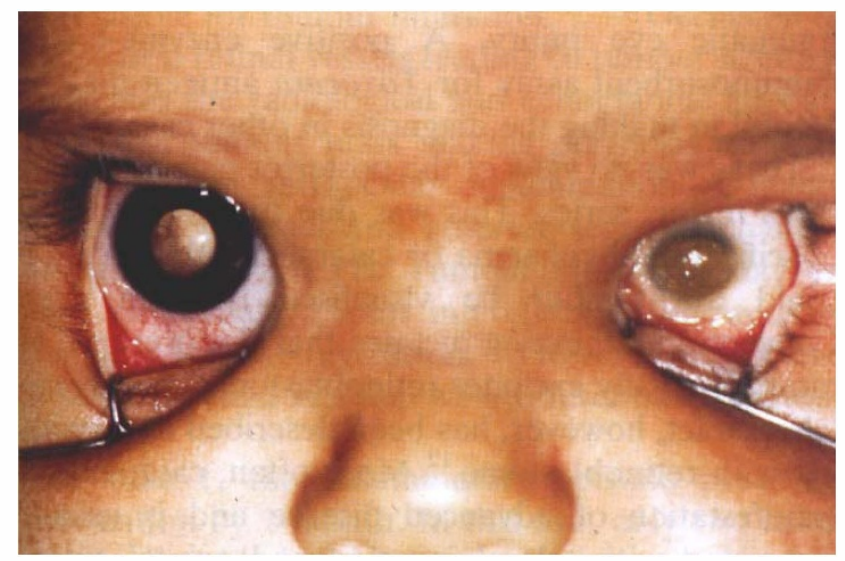

Fig. 2. Infant with bilateral retinoblastoma and left-sided $P B$.

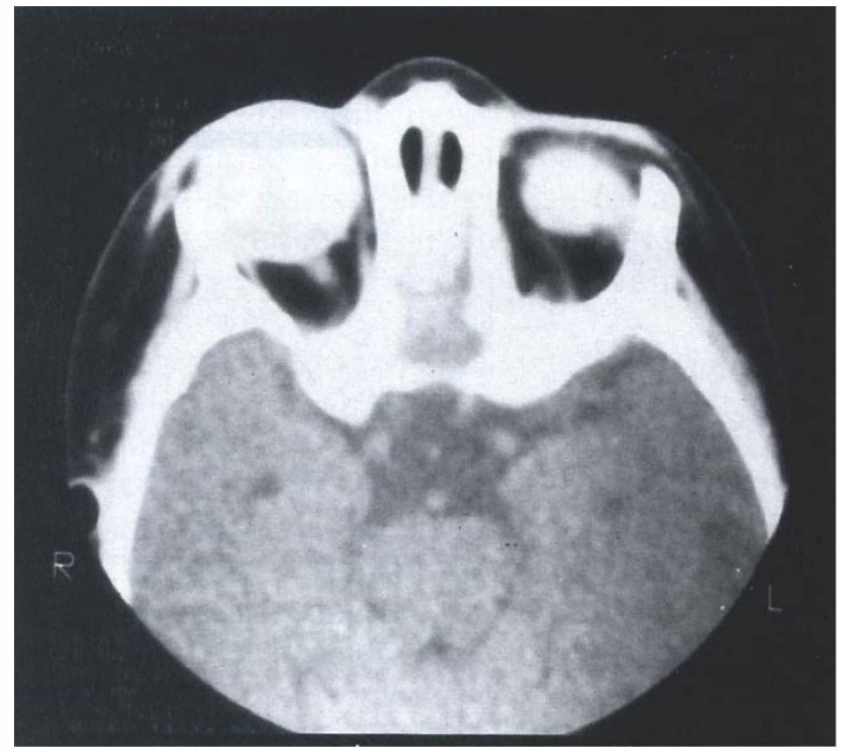

Fig. 3. CT scan of case 7 showing bilateral intraocular calcification and left-sided $P B$.

central part of the anterior chamber contained a whitish material which precluded any view of the posterior segment. CT scan confirmed a large calcified tumour in this eye (Fig. 3). The child underwent enucleation with implantation of a 16 mm scleral-wrapped 'baseball' implant.

Histopathological examination revealed the presence of a large, well-differentiated retinoblastoma occupying the entire globe with massive choroidal involvement. The tumour was very viable with only a small amount of necrosis and focal calcifications. Anteriorly, the tumour was abutting the iris-lens diaphragm. The lens revealed severe cataractous changes with focal areas of calcification. The architecture of the anterior segment was extensively distorted with diffuse thickening, fibrosis and vascularisation of the cornea, obliteration of the anterior chamber angle, and iris atrophy (Fig. 4). Diffuse rubeosis was present on the anterior surface of the residual iris and anterior lens capsule. A dense, partially calcified, fibrotic membrane was identified retrolentally between the tumour and the cataractous lens. No tumour cells were present within the anterior chamber. In addition to the phthisical changes seen anteriorly, irregular thickening and fibrosis of the sclera, disorganisation of the choroid with focal areas of fibrosis and calcification were present. The optic nerve had focal atrophy of its intrascleral segment with proliferation of subarachnoid cells secondary to its 'choking' due to phthisis. The pre-laminar part of the optic nerve was infiltrated with retinoblastoma cells, but no other evidence of optic nerve, scleral or extraocular tumour was seen. 

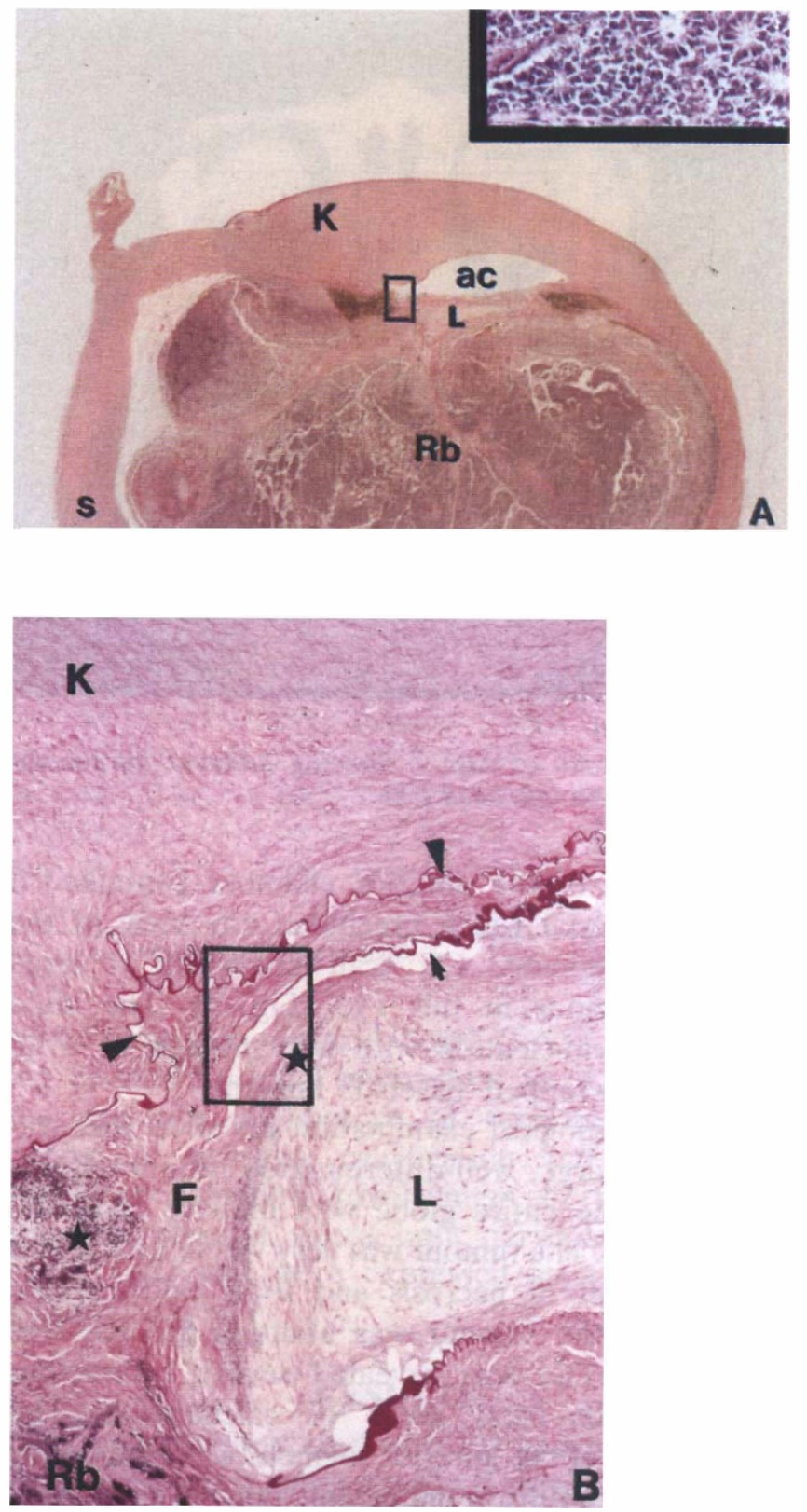

\section{DISCUSSION}

Phthisis bulbi is an atypical presentation of retinoblastoma. In this review, we found the incidence to be $2.7 \%$. This correlates well with other series, which found the incidence to be $2 \% .^{1,2,7}$ There was no common temporal pattern. Two patients died during this study: case 10 where enucleation was declined, and case 5 where extraocular extension occurred from the fellow eye.

When retinoblastoma presents with $\mathrm{PB}$, the clinical picture is often obscure. Trauma, endophthalmitis and uveitis are common causes of $\mathrm{PB} .{ }^{8,9} \mathrm{~A}$ history of trauma may help to rule out occult retinoblastoma though it must be remembered that retinoblastoma may present coincidentally as a
Fig. 4. (A) Low-power photomicrograph of the right eye of case 7 depicting extensive retinoblastoma associated with phthisical changes. (Inset: Higher magnification of retinoblastoma from phthisical eye, depicting well-differentiated histology. Original magnification: $\times 100$.) Cornea $(K)$ and sclera $(S)$ are irregularly thickened and vascularised. $(A)-(C)$ The architecture of the anterior segment is extensively distorted secondary to marked fibrosis $(F)$ with an extremely shallow residual anterior chamber (ac). Descemet's membrane (arrowheads) and the anterior lens capsule (arrows) are entrapped with fibrosis. Lens (L) reveals changes of end-stage cataract with dense fibrous ingrowth and areas of calcification (*). Partially calcified retinoblastoma cells $(R b)$ can be identified in the lower left of $(B)$. The boxed areas in $(A)$ and $(B)$ approximately correspond to $(B)$ and $(C)$ respectively. $(H \& E$; original magnifications: $\times 10(A), \times 100(B)$ and $\times 250(C)$.)

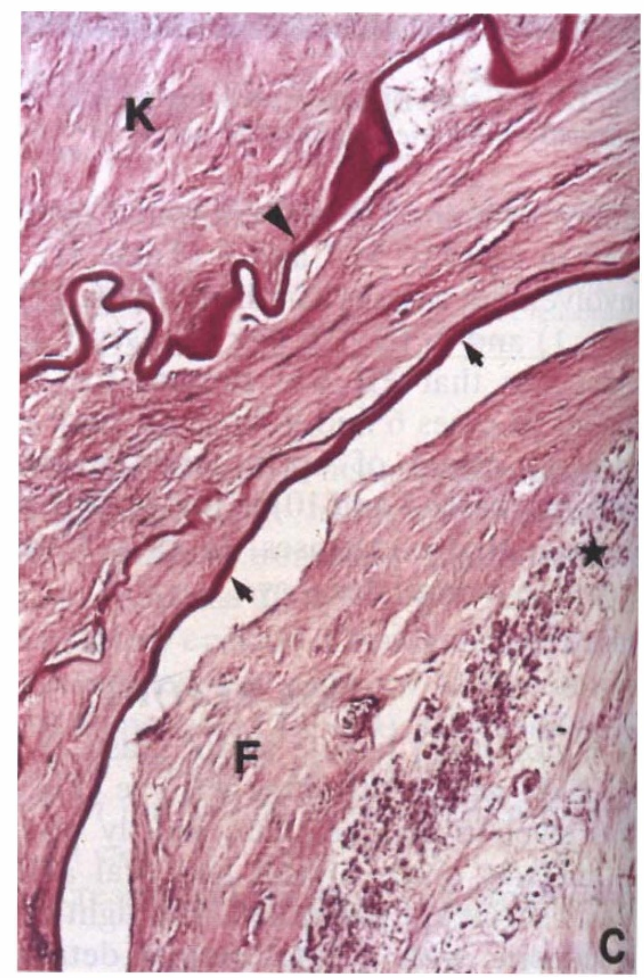

traumatic eye injury. A positive enzyme-linked immunosorbent assay for Toxocara antigen may be helpful in making the diagnosis in other cases. ${ }^{10}$

There was no view of the posterior pole in 9 of 10 cases in this series. This was due to either corneal opacification, hyphaema or cataract. Only in case 6 could the tumour be visualised in both eyes. Conventional thinking dictates that the presence of cataract usually excludes retinoblastoma. ${ }^{11}$ Congenital cataract, however, has been described as coexisting with retinoblastoma. ${ }^{12}$ More often, cataract is a manifestation of advanced disease and is usually associated with scleral invasion and orbital extension. ${ }^{13-15}$ Where retinoblastoma occurs bilaterally, the diagnosis may be made easier by the visualisation 
of tumour in the fellow eye. The combination of PB and contralateral buphthalmos is highly suggestive of bilateral retinoblastoma. 5

In unilateral $\mathrm{PB}$ the clinical diagnosis can be difficult. This is illustrated in case 10 , where no intraocular view was afforded. Both CT scan and ultrasound showed no mass lesion and minimal intraocular calcification. Endophthalmitis of a possible infective origin was thought to be the most likely cause of PB. Cases 1 and 2 were similar, though serial CT scans showed increasing intraocular calcification, thus providing the rationale for enucleation and avoiding the fatal outcome which occurred in case 10 . Where the diagnosis is in doubt, it is safest to enucleate a phthisical eye. When the case for enucleation cannot be made clearly, or this option is declined by the parents, patients should be followed-up with serial CT scans, which may show increasing intraocular calcification or extraocular involvement in later stages of the disease. Although magnetic resonance imaging (MRI) is less sensitive than CT for detection of calcification, its better soft tissue resolution allows superior views of the optic nerve which may help to rule out its invasion by retinoblastoma. ${ }^{16}$ The widening of the optic nerve in phthisical eyes containing retinoblastoma should be interpreted cautiously, since the enlargement may result not from retinoblastoma invasion but from the 'choking' effect on the nerve secondary to posterior scleral thickening of phthisis.

Intraocular infarction and concurrent tumour necrosis are thought to be pre-phthisical events. ${ }^{17.18}$ Panophthalmitis accompanied by preseptal and/or orbital cellulitis are thought to be secondary to tumour necrotic products leaching from the eye resulting in periocular inflammation. Parents of 7 of 8 patients specifically questioned were affirmative about episodes of periocular swelling. This would tend to offer evidence that episodes of intraocular infarction accompanied by inflammation precede PB. Central retinal vessel occlusion is thought to be responsible for tumour infarction. ${ }^{4,19,20}$ Occlusion of the central retinal vessels leading to tumour necrosis eradicates the radiological and echographic appearance of a tumour mass making diagnosis difficult. The resulting intraocular inflammation probably causes the cataract and anterior segment changes which are seen in PB. Tumour infarction may be total or partial. ${ }^{21}$ Total infarction is followed by complete tumour regression. ${ }^{20}$ However, every eye in our series had residual well-differentiated tumour cells.

In case 1 , the inflammatory episode preceded enucleation by 1 month. In this eye, only two small islands of viable-looking tumour cells were seen. It is conceivable that these had established ancillary blood supplies from the choroid or optic nerve. In cases 6 and 9 the extraocular extension of retinoblastoma was greatest; in case 9 it extended intracranially. In both these cases PB had been present for a long period of time, suggesting (as expected) that where $\mathrm{PB}$ is present for a long time, the risk of extraocular extension is greatest. This underlies the danger of not removing a phthisical eye of unknown origin.

Tumour necrosis was marked in all eyes, indicating a possible vaso-occlusive effect. However, marked calcification was present. It has been suggested that calcium has a toxic effect on retinoblastoma cells. ${ }^{18}$ There is some experimental evidence for this. ${ }^{22}$

Calcification in PB of non-neoplastic origin is described as affecting the ocular wall, in particular the choroid. ${ }^{23}$ Choroidal bone formation, often with haematopoietic elements, can be seen. This calcification appears to be different from that seen in retinoblastoma. In retinoblastoma the calcification involves the tumour which is occupying the central aspect of the globe. The difference sometimes can be apparent on CT scan - PB with a circular ring of calcification involving the ocular wall can be appreciated as opposed to the often centralised opacities seen in retinoblastoma. However, this distinction may be of little comfort to the clinician who is trying to decide on the appropriateness of enucleation.

In conclusion, this study confirms that $\mathrm{PB}$ is a rather rare presenting sign of retinoblastoma, but nevertheless can be encountered on occasion. The absence of clear clinical signs can often obscure the diagnosis. CT scan may show characteristic intraocular calcification or extraocular pathology. However, this is by no means conclusive. In view of the fact that this study found viable tumour cells in every eye examined, a high index of suspicion for retinoblastoma should be maintained in $\mathrm{PB}$ of unknown origin.

Key words: Calcification, Intraocular, Children, Enucleation, Optic nerve, Phthisis bulbi, Retinoblastoma.

\section{REFERENCES}

1. Gallie BL, Phillips RA, Ellsworth RM, Abramson DH. Significance of retinoma and phthisis bulbi for retinoblastoma. Ophthalmology 1982;12:1393-9.

2. Carbajal UM. Observations on retinoblastoma. Am J Ophthalmol 1958;45:391-402.

3. Manschot WA. Difficulties in the clinical diagnosis of retinoblastoma. Ophthalmologica 1956;132:162-4.

4. Andersen SR, Jensen OA. Retinoblastoma with necrosis of the central retinal artery and vein and partial spontaneous regression. Acta Ophthalmol (Copenh) 1974;52:183-93.

5. Boniuk M, Zimmerman LE. Spontaneous regression of retinoblastoma. Int Ophthalmol Clin 1962;2:525-42.

6. Hiatt R, Kendrick DL, DuPont G. Retinoblastoma: regression and progression. Am J Ophthalmol 1961;52: 717-23.

7. Sherman NS. Significance of phthisis bulbi in retinoblastoma. Am J Ophthalmol 1959;47:403-5. 
8. Brackup AB, Carter KD, Nerad JA, Folk JC, Pulido JS. Long-term follow-up of severely injured eyes following globe rupture. Ophthal Plast Reconstr Surg 1991;7:194-7.

9. Capone A Jr, Auberg TM. Intermediate uveitis. In: Albert DM, Jakobiec FA, editors. Principles and practice of ophthalmology, vol 1. Philadelphia: Saunders, 1994:423-31.

10. Albert DM, Marcus LC, Searl SS, Moazed K. Ocular toxocariasis presenting as leukocoria in a patient with low ELISA titer to Toxocara canis. Ophthalmology 1981;88:1302-6.

11. Shields JA, Shields CL. Differential diagnosis of retinoblastoma in intraocular tumours: a text and atlas. Philadelphia: Saunders, 1992:341-62.

12. Friendly DS, Parks MM. Concurrence of hereditary congenital cataracts and hereditary retinoblastoma. Arch Ophthalmol 1970;84:525-7.

13. Schuster SAD, Ferguson EC. Unusual presentations of retinoblastoma. South Med J 1970;63:4-8.

14. Ellsworth RM. The practical management of retinoblastoma. Trans Am Ophthalmol Soc 1969;67:462-534.

15. Kopelman JE, McLean IW, Rosenberg SH. Multivariate analysis of risk factors for metastasis in retinoblastoma treated by enucleation. Ophthalmology 1987;94:371-7.
16. Mafee MF, Greenwalt MJ, Goldberg MJ, et al. Retinoblastoma and simulating lesions: role of CT and MR imaging. Radiol Clin North Am 1987;25: 667-82.

17. Shields JA, Shields CL, Suvarnamani C, Schroeder RP, DePotter P. Retinoblastoma manifesting as orbital cellulitis. Am J Ophthalmol 1991;112:442-9.

18. Verhoeff FH. Retinoblastoma undergoing spontaneous regression: calcifying agent suggested in treatment of retinoblastoma. Am J Ophthalmol 1966;62:573-4.

19. Wolter JR. Thrombosis of the central retinal artery in retinoblastoma. J Pediatr Ophthalmol 1976;13:99-102.

20. Marcus DM, Craft JL, Albert DM. Histopathologic verification of Verhoeff's 1918 irradiation cure of retinoblastoma. Ophthalmology 1990;97:221-4.

21. Khodadoust AA, Roozitalab HM, Smith RE, Green WR. Spontaneous regression of retinoblastoma. Surv Ophthalmol 1977;21:467-78.

22. Cohen SM, Saulenas AM, Sullivan CR, Albert DM. Further studies of the effect of vitamin $D$ on retinoblastoma: inhibition with 1,25-dihydroxycholecalciferol. Arch Ophthalmol 1988;106:541-3.

23. Hogan MJ, Zimmerman LE. Diffuse ocular disease and its sequelae. In: Ophthalmic pathology: an atlas and textbook. Philadelphia: Saunders, 1962:96-136. 